\title{
TEM Observations of Nano-porous Hematite formed by Dehydration of Synthetic Goethite Particles
}

\author{
James H Steele Jr Consultant, 7412 Singing Hills Court, Boulder, CO 80301
}

The transformation of synthetic $\alpha$-goethite particles to nano-porous hematite occurs as a topotactic transformation, wherein the fcc oxygen ion lattice remains intact. The particles transform via $\mathrm{H}_{2} \mathrm{O}$ removal, which creates slit shaped nano-pores that alternate with twinned hematite crystallites. [1] The kinetics of the dehydration reaction, which depend upon the goethite particle size, are shown in Figure 1, via integrated peak intensity measurements from x-ray powder diffraction patterns. A mosaic bright field TEM image of the synthetic goethite crystallites whose kinetics were measured is presented as Figure 2. SAD patterns before and after in situ dehydration by electron beam heating of a single goethite particle are presented in Figure 3. This demonstrates that the overall size and shape of the particles is essentially unchanged by the dehydration process. The nano-pores formed during dehydration tend to coarsen rapidly with heat treatment at $483 \mathrm{C}$ or with continued electron beam heating, as illustrated in Figure 4.

\section{References:}

[1] Watari, et al, J. Solid State Chemistry, Vol 48 ( 1983 ) pp 49-64.

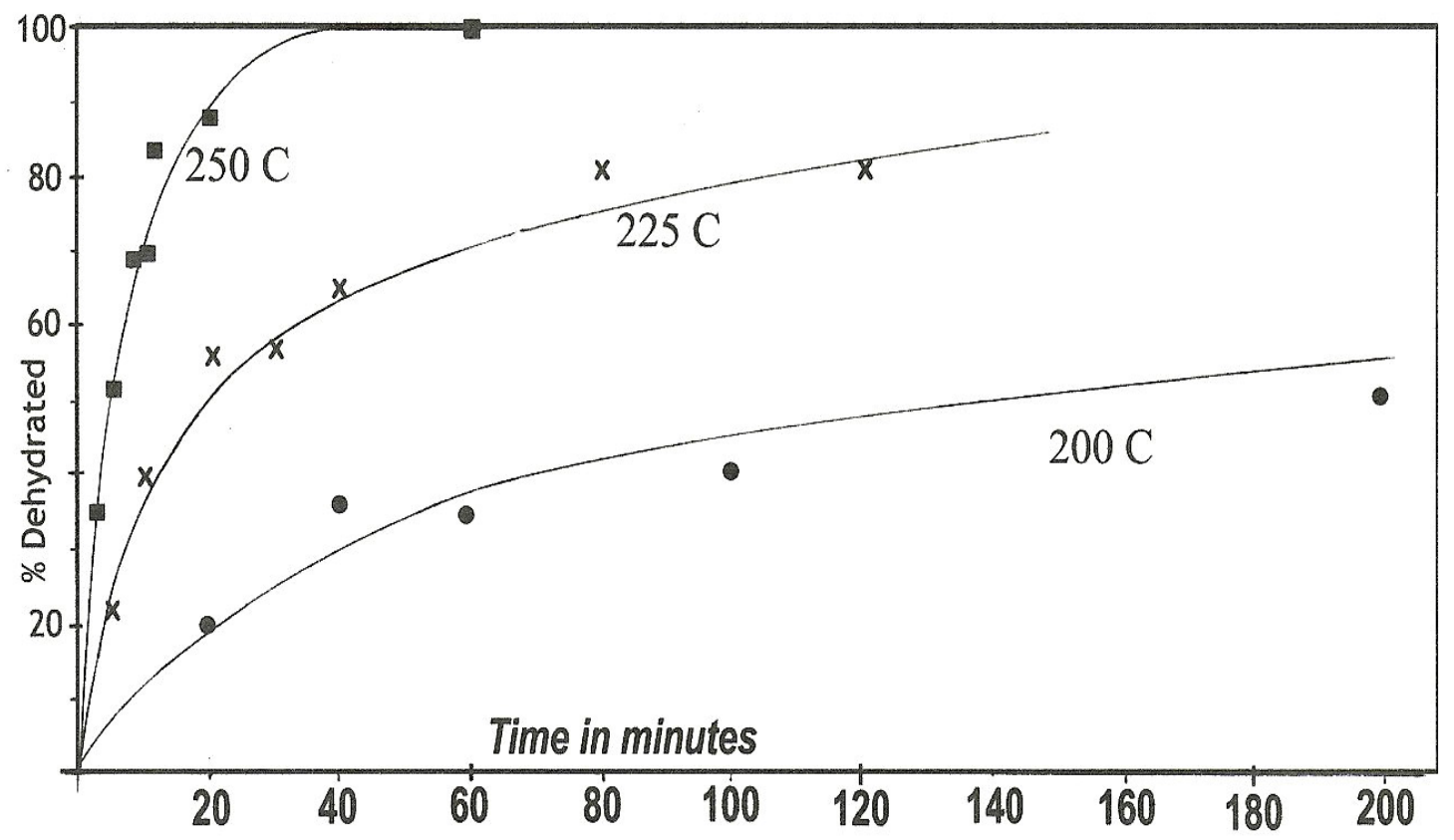

Figure 1 Dehydration kinetics of the synthetic Goethite particles illustrated in Fig. 2. 

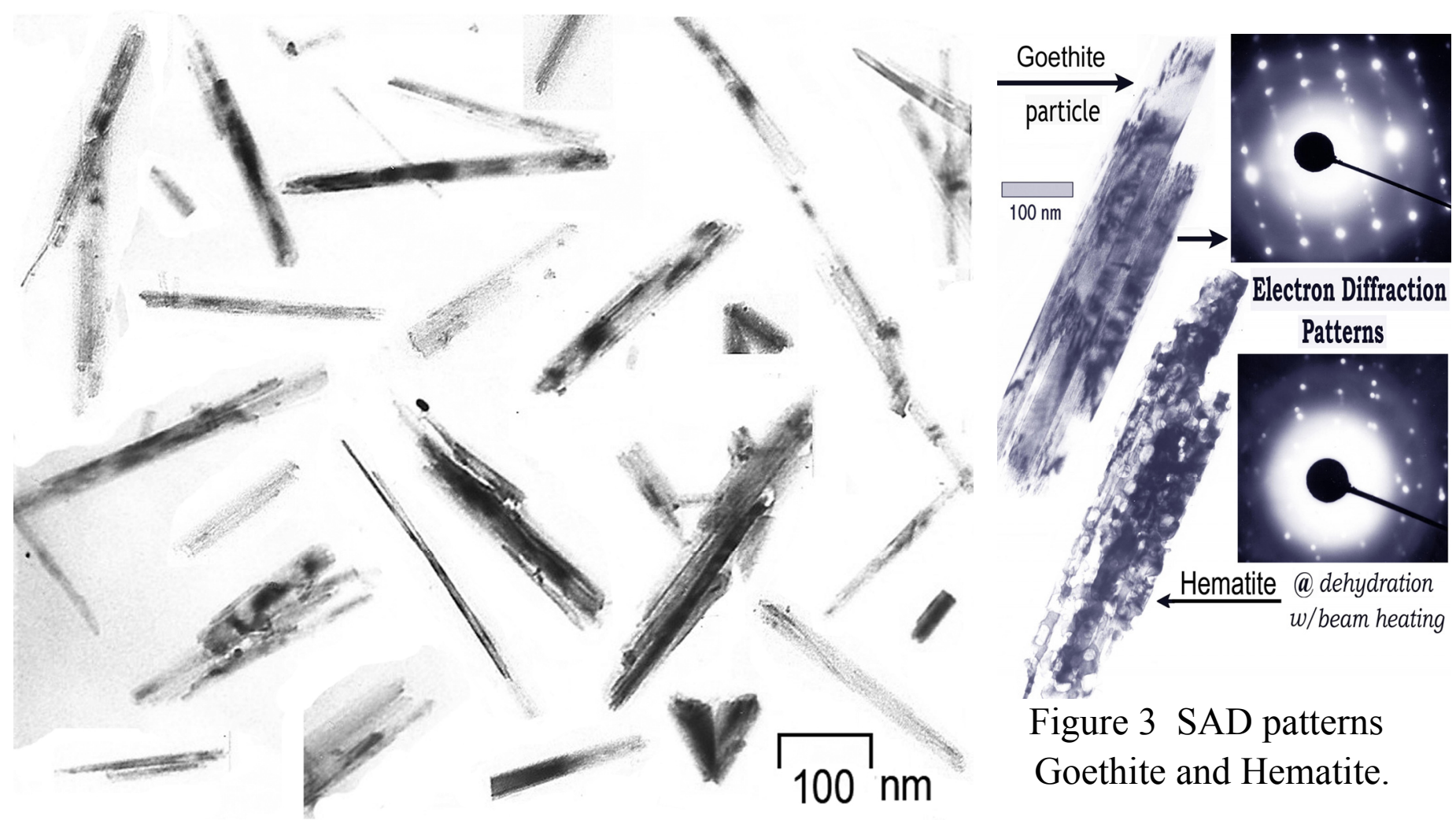

Figure 3 SAD patterns

Goethite and Hematite.

Figure 2 Bright field TEM of synthetic goethite particles.

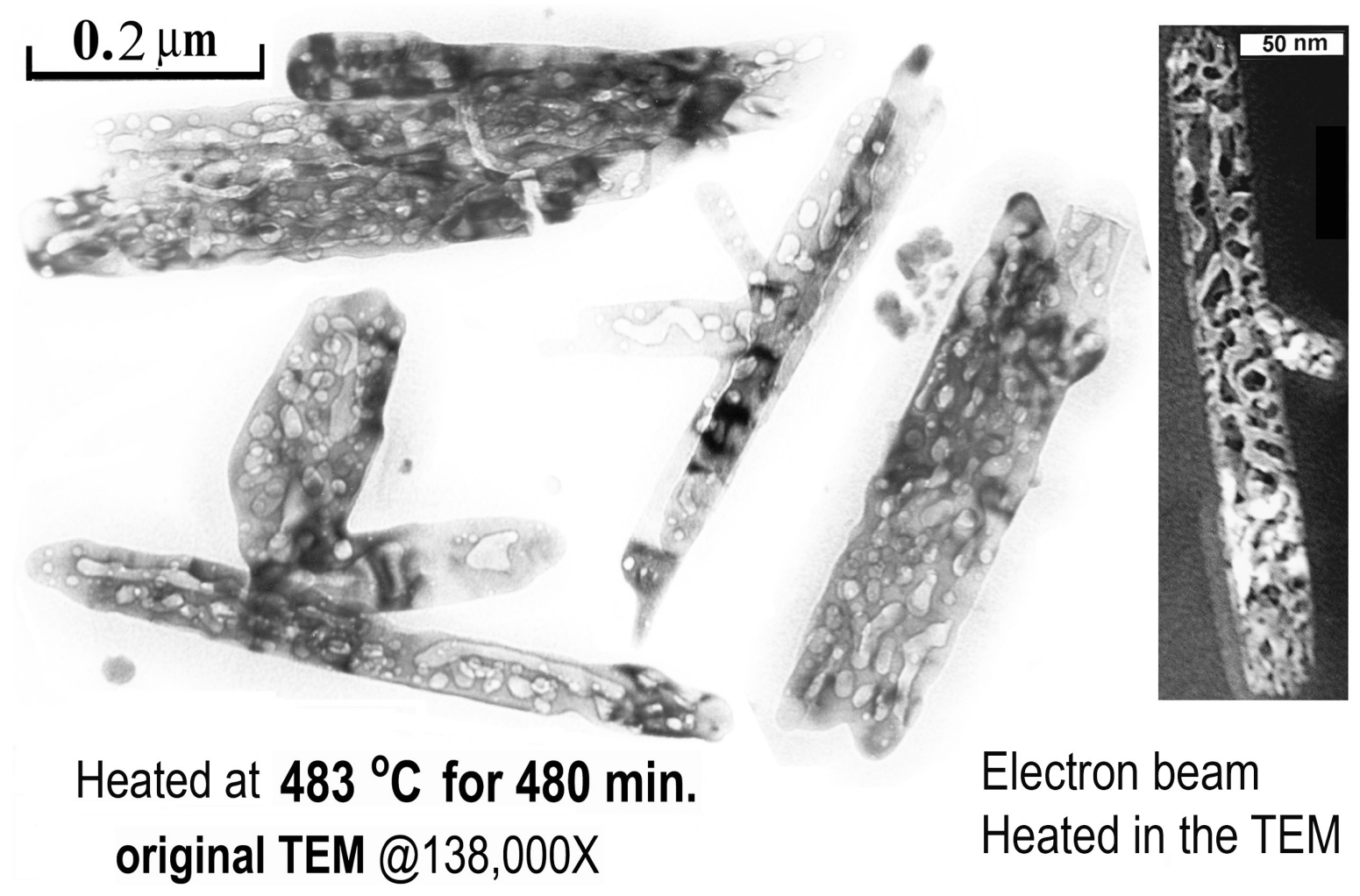

Figure 4 Bright field TEM micrographs of nano-porous Hematite particles. 